\title{
Image Guided Biopsy Procedures
}

In oncology practice pathological diagnosis of the disease process is of paramount importance and is always considered a gold standard for diagnosis. Ability of the fine needle aspiration cytology (FNAC) to provide diagnosis on examination of few cells and its safety profile has made it one of the most widely used diagnostic procedure in oncology.

With wide availability of radiological modalities like ultrasound and CT scan and emergence of the subspecialty of the interventional radiology, routine diagnostic surgical procedures are now uncommonly performed. Image guided percutaneous biopsies are minimally invasive, cheaper and can be performed with little post procedure observation of the patient, usually in a day care facility. These also do not require general anesthesia except in children or uncooperative patients. Life threatening complications of percutaneous biopsies are rare but not unknown.
Adequate surgical backup to deal with such rare but unforeseen events must be readily available.

Fluoroscopy guided percutaneous biopsies of the lung and bone lesions have been traditionally performed, however, the scope of the percutaneous biopsy has significantly been expanded with widespread availability of cross sectional imaging modalities; ultrasound and CT in particular. MRI and mammography guidance is also used in appropriate situations. One of the important limitations of the nuclear medicine techniques like Radionuclide scintigraphy and PET scan is that the guided procedures can not be performed with these modalities.

CT scan is the most popular guiding modality. Advantages of CT include-clear depiction of all anatomical details and practically any area of the body can be accessed. Complications, if any, are readily recognized on CT scan. However, CT guided biopsy is expensive, involves radiation and 
takes longer time to perform. Also, the needle is not advanced in real time i.e. multiple images are obtained after each movement of the needle to confirm. Ultrasound, on other hand, is cheaper and radiation free. The needle is advanced and sample obtained under real time visualization. It is also faster than $\mathrm{CT}$ guided procedure. However, ultrasound suffers form limitation of visualization in some areas such as intrapulmonary or bone lesions as well as deep seated abdominal; lesions obscured by overlying gaseous bowel. Ultrasound guided interventions also have a definite learning curve.

Both FNAC as well as core biopsies have advantages and disadvantages. FNACs are simple and safer but are more prone for false negative diagnosis. Availability of on site cytopathologist who can confirm the adequacy of the harvested specimen using fast stain techniques is important to decrease false negative or inconclusive results. ${ }^{1}$

Core biopsies are less susceptible to false negative or false positive diagnosis when compared with FNAC. These also provide histological and architectural information, which is important in subtyping of some malignancies.

Core biopsy samples are also more suitable for special tests like immunohistochemisrty and receptor analysis. Newer developments in core biopsy techniques like automated spring loaded biopsy guns and use of coaxial needle systems allows us to obtain multiple large core samples with single puncture without significant increase in complications. ${ }^{2}$

It should be remembered that sampling error is inherent to any biopsy procedure. To minimize the misdiagnoses biopsy results must be correlated with clinical, laboratory or radiological findings and in case of any discordance, the procedure must be repeated or open surgical biopsy be obtained.
Interventional radiology is a wellestablished subspecialty of the radiology. With improvement in the biopsy techniques, proven track record and safety profile and its acceptance both by referring clinicians and the performing radiologists, it is not surprising that image guided biopsy today is the most commonly performed interventional radiological procedure. ${ }^{4} \mathrm{~A}$ well-planned and executed image guided biopsy provides an accurate diagnosis and facilitates institution of the definitive treatment. An article on CT guided percutaneous biopsies of thoracic lesions published in this issue of the Journal highlights the increasing acceptance of this simple but useful diagnostic technique in oncology practice. ${ }^{5}$

\section{REFERENCES:}

1. Mullar DA, Carrasco CH, Katz RL, et al. FNAB: the role of immediate cytologic assessment American Journal of Roentgenology 1986;147:155-58.

2. Moullen IS, Merc PR. Percutaneous coaxial biopsy technique with automated biopsy needle - value in improving accuracy and negative predictive value. Radiology 1993;186:515-22.

3. Zinzani PL, Colecchia A, Fest D, et al. US guided core needle biopsy is effective in initial diagnosis of lymphoma. Hematologica 1998;83:989-92.

4. Mouton JS, Leoni CJ, Quarfardt SD, Woth $S$. Percutaneous image guided biopsy In Baum $S$, Pentcort $M$ (ed). Abrams Angiography: Interventional Radiology. $2^{\text {nd }}$ edition Lippincot William Wilkins. 2006. Philadelphia pp255-78.

5. Prashant, Ramachandra $C$ Pattbhiraman et al. Feasibility, Safety, and efficacy of the CT guided Fine needle aspiration cytology (FNAC) of lung lesions. Ind $J$ Med \& Paed Oncol 2007:28:16

Sanjay Thulkar Department of Radiodiagnosis Institute Rotary Cancer Hospital All India Institute of Medical Sciences New Delhi-29 